\title{
Three Dimensional Stochastic Reconfiguration of Modular Robots
}

\author{
Paul White* ${ }^{* \dagger}$, Victor Zykov ${ }^{\dagger}$, Josh Bongard ${ }^{\dagger}$, and Hod Lipson ${ }^{\dagger}$ \\ ${ }^{\dagger}$ Computational Synthesis Laboratory \\ Cornell University, Ithaca, NY 14853 \\ Email: [vz25 | josh.bongard | hod.lipson]@cornell.edu \\ *Lockheed Martin Corporation, King of Prussia, PA 19406 \\ Email: paul.j1.white@1mco.com
}

\begin{abstract}
Here we introduce one simulated and two physical three-dimensional stochastic modular robot systems, all capable of self-assembly and self-reconfiguration. We assume that individual units can only draw power when attached to the growing structure, and have no means of actuation. Instead they are subject to random motion induced by the surrounding medium when unattached. We present a simulation environment with a flexible scripting language that allows for parallel and serial selfassembly and self-reconfiguration processes. We explore factors that govern the rate of assembly and reconfiguration, and show that self-reconfiguration can be exploited to accelerate the assembly of a particular shape, as compared with static self-assembly. We then demonstrate the ability of two different physical three-dimensional stochastic modular robot systems to self-reconfigure in a fluid. The second physical implementation is only composed of technologies that could be scaled down to achieve stochastic self-assembly and self-reconfiguration at the microscale.
\end{abstract}

\section{INTRODUCTION}

Modular robotics - a robot that is composed of a variable number of homogenous, possibly detachable units-has become of great interest in the robotics community recently for a number of reasons, including economic mass production of units, graceful degradation of function when damaged, and the ability to transition into topologies suitable to the task at hand. Two additional benefits are the ability to self-assemble, which is to organize a multi-unit system from a collection of independent units, and self-reconfiguration, which is the ability to transition from one topology to another using a series of attachments and detachments. Self-repair, a special type of self-reconfiguration, could allow a robot to replace damaged units with fresh units, or to reconfigure into a different shape in order to continue with the task at hand.

One of the first modular robot systems, CEBOT, was developed by Fukuda and Kawauchi [1]. Yim initially developed a set of modular robots [2], and has since developed a series of self-reconfigurable robots that employ different locomotion strategies: one example of this latter type of robot is PolyBot [3]. Chirikjian developed a metaphoric robot system that could form structures by rolling over each other in a plane [4]. Rus and Vona [5] developed Cystalline, modules that can form robotic systems by collapsing and expanding the body of each module. Rus et al have also studied different forms of self-organizing robotics through their experiments with the prototypes of Molecules [6][7], modules that have a pair of two degree-of-freedom atoms and can successfully form 3D shapes. Murata et al originally developed the Fracta robot system [8][9], which can reconfigure by rotating units about each other. Tomita et al [10] have since extended this work to a system in which modules can climb over one another, and Yoshida et al [11] have developed a miniaturized selfreconfigurable robot. Ichikawa et al [12] have demonstrated a collective robotics system in which individual robots can attach and detach from each other to form variable multirobot structures. Støy et al [13] developed a role-based control strategy for the self-reconfigurable modular robot CONRO [14]. Lund et al are developing land-based [15] and waterbased [16] modular robot systems. The water-based system is still in its prototype phase, and although will be influenced by stochastic fluid forces, the individual units are designed to move independently and deterministically. For a review of modular robotics, see [17].

However in all of these approaches, it is assumed that the individual units have onboard power and the ability to locomote, or are deterministically moved to the appropriate place by other powered units. Such explicit reconfiguration and assembly processes offer many advantages, but also place severe power and mechanical actuation challenges on the design of each module. In particular, these requirements limit the scalability of such systems to smaller scales, where power storage is difficult and mechanical locomotion and actuation possibilities are limited.

The robot units described in this paper are at root stochastic: units have no independent means of power or locomotion, and rely on simulated Brownian motion induced by agitation of the surrounding medium for locomotion; the units themselves are passive, and only draw power when captured and anchored to the growing system. This form of reconfiguration avoids many of the barriers that prevent self-reconfigurable robotics from extending to large numbers and small scales. As scales reduce, deterministic active locomotion becomes increasingly difficult, whereas stochastic passive motion becomes easier. Biological and physical systems at the micro- and nano-scales rely extensively on such parallel stochastic self-assembly and reconfiguration based on passive motion, and this tendency is progressively pronounced as scales decrease. To date, artificial 
systems based on stochastic self-assembly of structures have been demonstrated at the millimeter scale [18] and at the nanoscale [19], but are not reconfigurable: they assemble-like a puzzle-according to predesigned templates. This approach is also difficult to scale to complex, non-periodic, arbitrary 3D configurations and is very sensitive to matching errors.

Klavins et al [20] have begun exploring a grammatical approach to controlling the stochastic self-assembly of structures. They have demonstrated its application using 2D triangleshaped tiles [21], though their built-in power supply and permanent magnets prevent this approach from being implemented at the microscale. The work reported here improves on the 2D system introduced in [22] by introducing one physical system comprised of technology that could also conceivably work at the microscale.

While the underlying process we propose is stochastic, we still wish to deterministically control the overall global configuration and behavior of the system, and so new structural and control issues must be addressed. In this paper we present a three-dimensional physical simulation environment, along with a scripting language, with which we can explore these issues. We describe this system and some new results regarding selfassembly and reconfiguration in section III. In section IV we present the first simple, physical, three dimensional stochastic robot system that can self-assemble and self-reconfigure. We conclude in section V.

\section{THE CONCEPT}

A stochastic robotic system reconfigures inside an environment that enables Brownian motion. At the macro-scale, such environments exist in zero-gravity space or in agitated fluid surroundings, which simulates Brownian motion extant at smaller scales. At the micro- and nano-scales, almost any fluid environment facilitates such motion. After or between reconfigurations, a system may operate outside of this kind of environment.

Units of a stochastic modular robotic system have the following characteristics:

- Units are unpowered, and become active only once they bond to the main structure.

- Units have no locomotion ability. The potential location of a unit is determined by active bonding sites and the statistical mechanics of Brownian motion (or a simulated analogue).

- Unit interfaces are identical, and their function differentiates depending on their final context.

Structures can reconfigure by activating new bonding sites on their exterior and waiting for a floating unit to bond, or by rejecting connected units into the environment. (In the physical system described in section IV, units to one another by passive magnets on the units' faces, while the central electromagnet in each face, when activated, rejects attached units.) The likelihood of bonding depends on statistical mechanics properties of the motion and attraction basin of active bonding sites, along with the local geometry of the solid structure near the bonding site. Depending on the specific design of individual
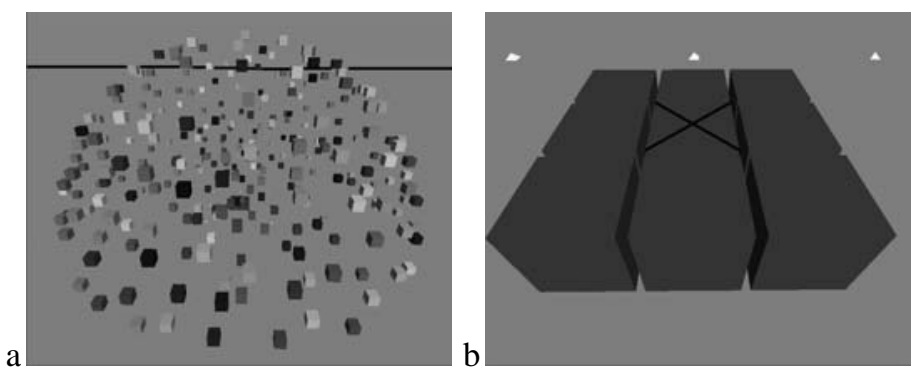

Fig. 1. The experimental enclosure for simulated experimentation with self assembly and reconfiguration. a: The enclosure. b: Magnification of the seed plate. The cross indicates that the top face of the middle block is magnetized.

modules, they can share power and information and cooperate to achieve global sensing, actuation and computation. Power for this system comes from the substrate on which it is grown, or from the environment in which it is embedded.

\section{Simulated Self Assembly and Reconfiguration}

We have developed a three-dimensional dynamical simulator in order to explore the issues involved in three-dimensional stochastic self assembly and reconfiguration. This simulation is supplemented with a simple scripting language that allows for rapid experimentation with different assembly and reconfiguration strategies, as well as different target geometries. The following two subsections describe the simulator and the scripting language, respectively. Subsection III-C investigates one factor that influences the time to completion for a given geometry: the use of reconfiguration as a scaffolding tool.

\section{A. The Simulator}

Our modular robot simulator is built on top of Open Dynamics Engine ${ }^{1}$, a three-dimensional dynamical simulator originally developed for the simulation of rigid articulated mechanical structures. However the base simulator was readily adapted for the simulation of multiple, free floating bodies. Figure 1a gives an overview of the modular robot arena. There are a total of 300 cubical blocks, each with a length of $20 \mathrm{~cm}$ and a mass of $1 \mathrm{~kg}$. Although the blocks are somewhat larger and heavier than the physical blocks described in the next section, the particularities of the simulation favor larger objects for the resolution of collision detection and resolution and high speeds. The blocks are contained within an inverted hemisphere, which contains an active base plate in the center of the hemisphere. The hemisphere has a radius of $5 \mathrm{~m}$. The total volume of the hemisphere is therefore $261.8 \mathrm{~m}^{3}$, and the total volume occupied by the blocks is $2.4 \mathrm{~m}^{3}$, such that the blocks occupy $0.9 \%$ of the total volume of the enclosure.

The blocks are assumed to be immersed in some fluid, and derive their linear velocity and acceleration from the action of this fluid; linear and rotational acceleration is also changed by collision with other blocks, the ground plane and the shell of the (invisible) hemisphere. Due to the heavy computational requirements of simulating hydrodynamics, the blocks were

\footnotetext{
${ }^{1}$ http://ode.org/
} 
agitated using a simpler process. At each time step $^{2}$ of the simulation, each block is perturbed by a small impulse force somewhere on the surface of the block. The position on the surface is chosen at random. The magnitude of the impulse force is also chosen at random, but the force is applied against the block at the chosen point inward toward the center of mass of the block. In this way, fluid agitation only induces linear velocity and not rotational velocity; blocks assume rotational velocity only after collision with other objects. This strategy is loosely based on what is observed for objects suspended in fluid exhibiting laminar rather than turbulent flow. When blocks or a block and the ground plane collide, inelastic collision is used to compute the resulting forces. When a block moves outside the radius of the enclosure, elastic collision is used to gently accelerate the block back in toward the enclosure.

The bottom of the enclosure also includes a $3 \times 3$ array of embedded blocks whose upper faces are exposed into the simulated fluid, and together act as the seed point for the assembly and reconfiguration of the structures (see Figure 1c).

It is assumed that blocks can magnetize one of their six faces when they are either part of the base plate, attached to the base plate or attached to an immobile structure growing from the base plate: i.e., blocks cannot exert an attractive force on free floating blocks unless they have been anchored to the structure and are receiving power from it. In future studies we intend to analyze systems in which one to five faces can be magnetized simultaneously (the sixth is assumed to be the anchor to the structure). Magnetic attraction is simulated as follows: when the face of a floating block comes within $20 \mathrm{~cm}$ of the magnetized face of an anchored block, a continuous force 'pulls' that face of the free floating block toward the magnetized face. If the distance between the faces exceeds $20 \mathrm{~cm}$, the force is canceled (to simulate the intermittent attraction between an anchored block and fast moving free floating blocks). If the distance between the faces closes to $10 \mathrm{~cm}$, four additional force vectors pull the four corners of the two faces toward each other, such that the free floating block approaches and aligns with the anchored block. If the distance between the faces closes to $0.1 \mathrm{~cm}$, the free floating block is anchored.

\section{B. The Scripting Language}

Three-dimensional structures can be assembled within the enclosure by repeatedly magnetizing selected faces of anchored blocks, capturing flee floating blocks, and assigning identities to the captured blocks. Many faces can be magnetized within the growing structure in parallel, but for the moment we assume that a single block can magnetize at most one face at a time. By specifying which blocks should be magnetized, and what should happen to a free floating block when it is captured, it is possible to indirectly specify the self assembly of a wide range of three-dimensional shapes. By further specifying that certain blocks should be released

\footnotetext{
${ }^{2}$ The simulation proceeds in discrete time.
}

in certain situations, it is possible to also achieve self reconfiguration: the loss of anchored blocks and the capture of new blocks can change one desired shape into another desired shape. To this end, we have developed a simple scripting language that allows the user-or, in future work, an automated process - to intuitively generate a list of events that, when executed in parallel or in series by the anchored blocks, achieves a particular series of three-dimensional shapes with high probability.

A particular script is composed of one or more sequential building stages. Each stage itself contains a series of events, where an event may be either the attraction and capture of a free block, or the jettisoning of an anchored block from the structure. Events within a construction phase may occur in series or in parallel. We further assume that each anchored block possesses a copy of the script to be executed, as well as a unique identifier. The process of assembly and reconfiguration begins by assigning identifiers to one or more of the blocks in the seed plate.

As an example, consider the construction process illustrated in Figure 2a-b. This process consists of two phases: the first produces the shape shown in Figure $2 \mathrm{a}$ and the second reconfigures this shape into the shape shown in Figure $2 b$. The first phase requires seven events to occur before the process can continue to the second phase. First, the four corner blocks are labeled as $A, B, C$ and $D$. The four upper faces of these blocks are then magnetized, and whichever free floating blocks are captured are given identifiers $E, F, G$ and $H$, respectively. These four events can occur in parallel. However, block $E$ cannot capture and label a free floating block with the identifier $I$ until it has itself been captured and identified by block $A$ (see Figure 2a). Therefore, the fifth event must wait for the first event to complete.

This approach to self assembly allows for a minimum of global communication: because each block contains a copy of the construction process and its own identifier, it only has to perform its own event. There is no need for a central authority to settle identifier arbitration, and a block does not have to wait on a signal from a distant block before executing its own event. The only time that inter-block communication is required is when a terminal block is anchored: a terminal block is one that does not magnetize one of its faces when captured. When a terminal block is captured it sends a message back along its parent chain (because there are no loops possible at the moment, there is no danger of the message getting lost); when the seed plate receives signals from all of the terminal blocks for the current phase, the phase is determined to have completed successfully. In the example shown in Figure 2a, the phase terminates when signals are received from terminal blocks $I, J, K$ and $L$. If a predetermined amount of time has passed and not all of the terminal blocks have reported in, the phase is determined to have terminated unsuccessfully. When a phase terminates correctly, the next construction phase can begin.

In the second and subsequent construction phases, blocks can be released from the structure, as well as captured. For 
TABLE I

Relative PERFormanCE FOR DifFEREnt STRATEgIES

\begin{tabular}{|r|r|r|}
\hline & Assembly & Reconfiguration \\
\hline \hline Construction Phases & 2 & 5 \\
\hline Total Units Involved & 10 & 15 \\
\hline Mean Time To Completion & $6611( \pm 798)$ & $5158( \pm 294)$ \\
\hline Min Time to Completion & 2948 & 2826 \\
\hline Max Time to Completion & 14496 & 8312 \\
\hline
\end{tabular}

example in Figure 2d, the two anchored blocks $D$ and $E$ are released. It is assumed that the block to be released signals its parent block to switch off its magnetized plate, thereby setting the block free. Once free, it again begins to move in response to external agitation and object collision. By releasing and capturing new blocks, the shape produced by one stage can be transformed into a new shape. In the next section we investigate how reconfiguration can aid in the construction of structures which are difficult to achieve only through iterative assembly.

\section{Effect of Reconfiguration on Time to Completion}

In the statistical mechanics of physical structures, such as crystals, atoms are only bonded to one another probabilistically, rather than permanently and deterministically. The building blocks of the structure are more or less free to rearrange themselves to minimize the energy in the system, in order to fill any internal cavities and relieve dislocations. As we have shown in a previous paper [22], deterministic bonding coupled with stochastic motion can in some cases prohibit the growth of complete structures: adding units at inopportune points in the construction process can hamper the addition of further units. As an extreme case, the growth of a filled sphere may be hampered by the chance construction of most of the shell before the core is built: free blocks may have little or no chance of moving into the interior of the sphere. Previously we have cited four factors that can influence the rate of structure formation: the density of free units; the energy of free units and the magnitude of agitation in the external fluid; the attraction strength of the bonding sites and the retention strength of the bonding mechanism. In this paper we identify a fifth and sixth factor, which is the method of construction, and the target structure itself, respectively.

In order to investigate the role that reconfiguration can play in the construction process, we formulated two alternative construction processes that result in a simple, five tower configuration (Figures $2 \mathrm{~b}$ and $\mathrm{g}$ ). The first process requires two construction phases, and does not involve the release of any anchored blocks during the process (Figures 2a-b). The first phase requires the bonding of eight blocks; the second phase requires the bonding of two blocks. The second process requires five phases, and does include the removal of anchored blocks (Figures 2c-g). This phase requires a total of 15 attraction and bondings, and five removals.

A total of 100 independent runs were performed: 50 used the first process and 50 used the second process. Each run begins with 300 free blocks distributed in the same uniform pattern throughout enclosure, but the random impulses and

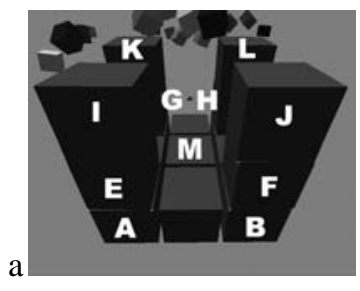

BlockA attracts BlockE above. BlockB attracts BlockF above. BlockC attracts BlockG above. BlockD attracts BlockH above. BlockE attracts BlockI above. BlockF attracts BlockJ above. BlockG attracts BlockK above. BlockH attracts BlockL above.

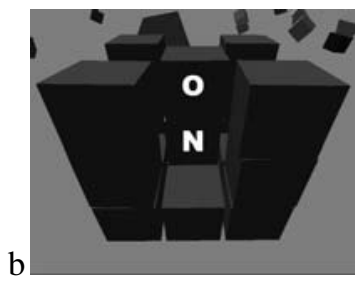

BlockM attracts BlockN above. BlockN attracts BlockO above.
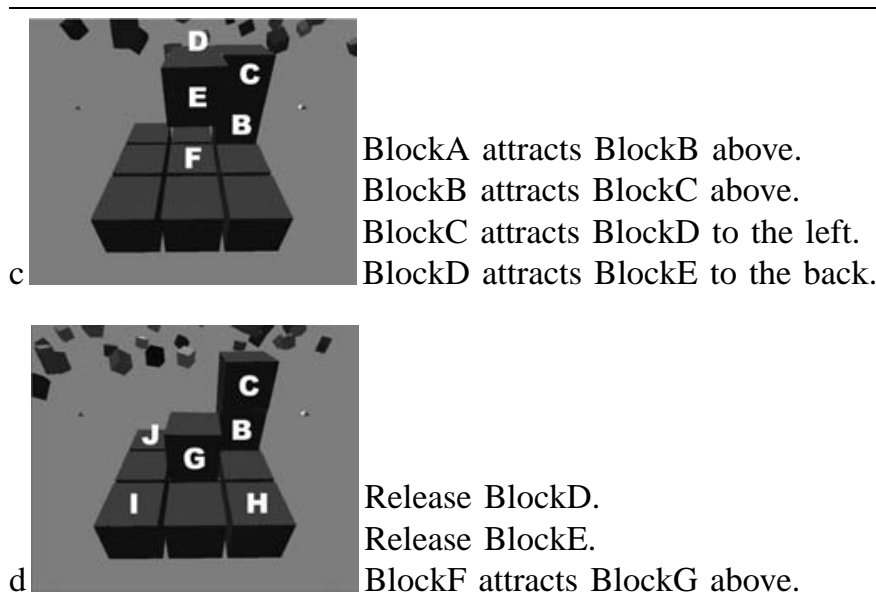

Release BlockD.

Release BlockE.

BlockF attracts BlockG above.

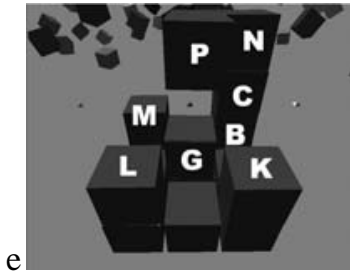

BlockH attracts BlockK above. BlockI attracts BlockL above. BlockJ attracts BlockM above. BlockC attracts BLockN above. BlockN attracts BlockO to the left. BlockO attracts BlockP to the back.
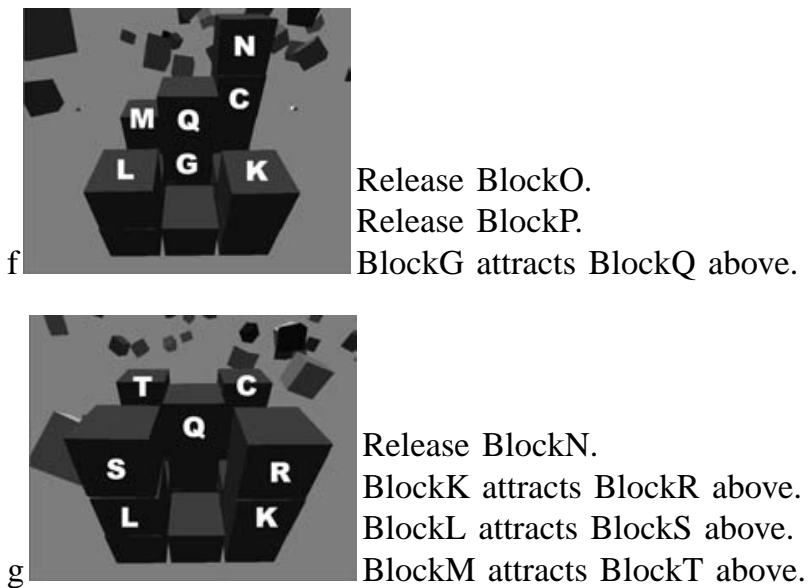

Fig. 2. Alternative construction strategies for the same shape. a-b: Strategy involving two construction phases. c-g: Strategy involving five phases Each image shows the final configuration after that particular construction phase. Some of the blocks are not labeled for the sake of clarity. 
collisions rapidly lead to diverging trajectories for the blocks between runs. Due to the stochastic motion of the blocks, each run requires more or less time to complete the entire construction process (each new phase is initiated in the time step immediately following the bonding of the final terminal block from the previous phase). The run terminates when the final terminal block of the final phase is anchored, and elapsed number of time steps is recorded. All of the 100 runs terminated correctly.

Table I reports that although the second strategy requires more construction phases and requires more bondings and releases, the second process tends to require less time than the first process, on average ${ }^{3}$. The reason that the first process takes so long is that an interior cavity forms: when the second phase begins, it takes a long time for a free block to move between the four corner towers in order to be captured by the magnetized upper face of the middle block (block $M$ in Figure 2a). Because of this, it takes the second phase much longer to complete than the first phase, even though the first phase requires eight bondings, while the second phase only requires two. For the slowest run of the first process, which required 14496 time steps, the first phase terminated after only 1499, while the second phase required a further 12997 time steps to capture the last two blocks.

The second construction process is faster than the first due to the use of reconfiguration: the two 'arms' that are constructed in phases one and three position blocks close to the upper face of the center block that will be magnetized in the subsequent phase. For example when phase three begins, the upper face of block $F$ tends to capture either block $D$ or $E$, which are both released during this phase (block $E$ is captured more often than block $D$ due to its proximity to block $F$ ). Released blocks are desirable for capture for two reasons: they are close to the growing structure, and they have little linear or angular velocity (as long as the release mechanism does not propel them away too violently). Because of this, intelligent sequences of shape reconfiguration can actually speed the construction of a target shape compared to if the shape is simply built from scratch.

In a parallel assembly task, of which robotic self-assembly is one example, the total amount of time required to complete the entire assembly is correlated with the time required for the longest set of serial processes ${ }^{4}$. In the case of the first assembly process, the longest serial process is two: the construction of any of the five short towers. The second assembly process requires one serial process of length four: the construction of the first arm during the first phase of construction (Figure 2a). Again, this would seem to indicate that the second process will take longer than the first, which is not the case due to the scaffolding effect of the constructed arms: the arms facilitate the capture of blocks above the center of the seed plate.

\footnotetext{
${ }^{3}$ Error intervals reported in this paper indicate standard error with a confidence level of $95 \%$.

${ }^{4}$ This is formalized in graph theory as the diameter of a graph, which is defined as the longest of the shortest paths between each pair of vertices in the graph.
}
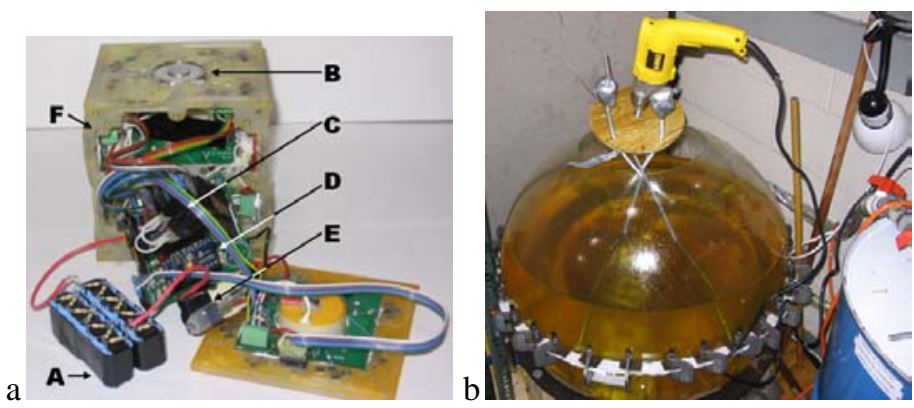

Fig. 3. A physical unit and the experimental tank. aA: 2/7 Farad capacitors. aB: Electromagnet. aC: Power, ground, magnet, side sensor, data transfer (6). aD: Basic stamp II. aE: Power resistor (sensing load). aF: Threaded brass inserts (12) and groove for sealant. b: The experimental tank and agitation apparatus.

\section{Physical Self Assembly and Reconfiguration}

In this section we introduce two physical, three-dimensional stochastic robot systems: the first employs magnets for bonding; the second employs fluid flow for bonding.

\section{A. Bonding Using Magnets}

We have developed, built and tested the first physical, threedimensional stochastic robot system, as shown in Figure 3. Figure 3a shows an exploded view of one of the cubical units, and Figure $3 \mathrm{~b}$ shows the spherical experimental tank in which the cubes were immersed. In this system all of the units are identical, and are neutrally buoyant ${ }^{5}$ when immersed in vegetable oil. The tank was filled with 150L of oil, and the bottom of the tank contains a magnetized plate which the units can attach to and draw power from. The plate has the same topology as the faces of the units, except that it only contains passive magnets. The four rods of the agitation apparatus shown in Figure $3 \mathrm{~b}$ are attached to a ring (not shown) with vertical teeth which is immersed in the fluid, and spins when the power tool is activated. This allows for the introduction of relatively constant rotational agitation of the oil by stirring. This agitation method serves as an analogue of Brownian motion at the macro scale: the units themselves are not powered and cannot move on their own.

The units themselves are $10 \times 10 \times 10 \mathrm{~cm}$ cubes, constructed from plastic with a set of magnets embedded in each of their faces. Sealing is achieved using a latex/silicon caulk, which is also used to seal the PCBS on the inner sides of the faces. Each face contains a series of bumps and indentations; passive magnets alternate in polarity radially in the same pattern as these bumps and indentations. At the center of each face is a switchable electromagnet. This face topology and magnet arrangement allows switchable bonding/rejection, and passive alignment: when two faces are attracted, the passive magnets align the faces, and the interlocking bumps and depressions cancel any relative rotation between the cubes that may occur due to external forces or the collision of free floating blocks. Experimentation has proven that a passive bonding method can

\footnotetext{
${ }^{5}$ In fact the units are just slightly denser than required for perfect neutral buoyancy so that they slowly settle near to the bottom of the tank and the magnetized plate.
} 
be a fatal design flaw if not accounted for. More precisely, the units have switchable rejection and continuous attraction.

When an experimental run begins, a unit may either be attached to the magnetized plate, or left to float freely in the fluid. If a unit is attached to the plate, or attaches to a structure in which one unit is attached to the plate, then the unit is anchored: it draws power from the plate (or through the structure from the plate). Each unit contains a set of instructions which direct that unit's behavior. The units contain a current sensor in each face, so that when a unit attaches and draws current from a particular face, the parent unit can determine which faces is anchoring the attached unit.

Instructions can be set such that when a face has anchored a unit for a period of time it can activate the electromagnet in that face. This imparts an impulse force to the unit to be rejected. Because of this rejection method, the force of the fluid, which is proportional to the velocity of the robot, must be accounted for. In addition, when two robots begin to separate, fluid needs to fill in the volume the robots create between them. Experimentation has shown that inadequate channels in the face of a robot can make separation difficult.

Two sets of experiments were carried out: in the first set a unit is first attached to the base plate; in the second set all units begin by floating freely in the oil.

1) Experiment I: Self-Assembly and Self-Reconfiguration: Fifty independent runs were carried out using a different agitation apparatus than that shown in Figure 3b: 5 plastic tubes (not shown) arranged along the inner walls of the partly filled tank and submerged in the oil introduced about $94.5 \mathrm{~L} / \mathrm{min}$ of more vegetable oil into the tank, thereby initiating mild agitation of the fluid. One unit was attached to the magnetized plate, and one of its side faces was magnetized. A second unit is introduced into the fluid near the inner periphery of the tank. A process of polarity switches on the anchored unit allowed for self-assembly and self-reconfiguration, as shown in Figure 4. Of the fifty runs that were performed, 12 successfully produced this self-assembly and reconfiguration pattern (giving a $24 \%$ success rate), indicating that much more research into maximizing the likelihood of assembly and reconfiguration needs to be conducted.

2) Experiment II: Passive Aggregation: One of the major factors affecting bonding and rejection is the energy of the system, or how units move in response to external forces. In order to test how agitation affects bonding in our system, the agitation apparatus shown in Figure 1b replaced the tube system for agitation. Three levels of agitation were achieved, where agitation was measured in terms of power introduced to the system, and power is calculated as

$$
P=F \times 2 \pi r \times \mathrm{rev} / \mathrm{s},
$$

where $F$ is the force applied by the four rods (together) to the agitation ring, tangential to its direction of rotation. The radius of the ring is $0.085 \mathrm{~m}$, and by increasing the power of the power tool the rotational speed of the ring can be increased to increase the agitation of the fluid.

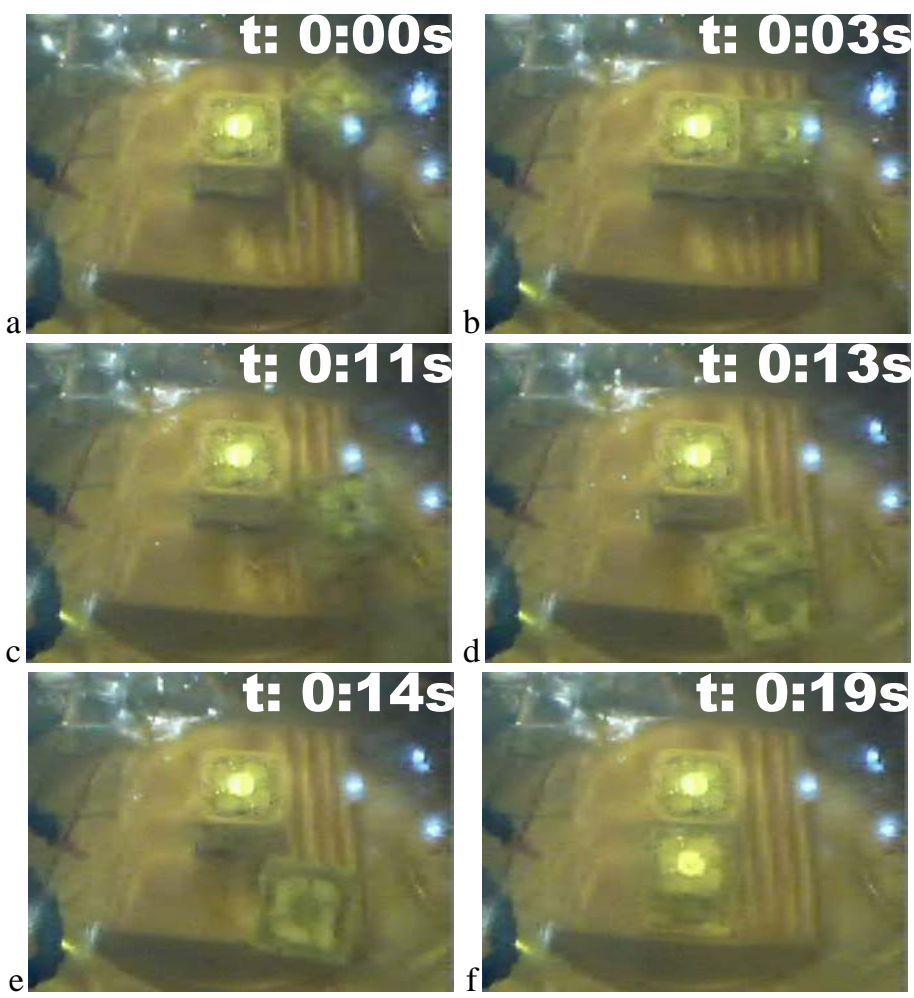

Fig. 4. Physical self-assembly and reconfiguration using magnets for bonding. The anchored unit attracts a free-floating block (a), which then attaches and bonds to the anchored block, demonstrating self-assembly of a two-unit structure (b). The second unit is then rejected, but the rejection, in addition to random fluid agitation, brings the unit close to a different face (c-e). The unit then re-attaches, resulting in a new two-unit structure, thereby demonstrating self-reconfiguration (f).

A total of 40 runs using this regime were performed. In the first 10 , the average rotational speed was $0.454 \mathrm{rev} / \mathrm{s}$, giving a power to the system of $0.193 \mathrm{~W}$. Two units were introduced into the tank diametrically opposite from each other. A further 10 runs were then performed using the same power input, but three separate units were placed in the tank, forming an imaginary equilateral triangle. A third set of 10 runs were then performed again with the same power, but with four separate units placed in the tank, forming a square. Table II indicates that the total time required to form a two-, three- or fourunit structure took on average $31.7,11.1$ and 6.9 seconds, respectively. A fifth and six set of experiments were performed using the two-unit setup: in the first set of 10 runs power was increased to $0.283 \mathrm{~W}$; in the sixth set of 10 runs power was increased to $0.472 \mathrm{~W}$. For these cases the mean time to bond was $14.9 \mathrm{~s}$, and never; in this last regime, the force of fluid agitation overpowered any initial magnetic attraction between the units.

As Table II makes clear, the average time to bond increases with the density of units. Also, there is some agitation energy (which is somewhere between $0.193 \mathrm{~W}$ and $0.472 \mathrm{~W}$ ) for which mean time to bonding is minimized, as evidenced by the faster speed with which units bond in the intermediate level of energy compared to the speed of bonding in both the low and high energy regimes. 
TABLE II

Passive Aggregation Versus Energy

\begin{tabular}{|r|c|c|c|}
\hline Energy & Low & Medium & High \\
\hline \hline rev/s & 0.455 & 0.667 & 1.111 \\
\hline Power (W) & 0.193 & 0.283 & 0.472 \\
\hline $\begin{array}{r}\text { Time to Assemble } \\
\text { Two Units }\end{array}$ & $\begin{array}{c}31.7 \\
( \pm 11.34)\end{array}$ & $\begin{array}{c}14.9 \\
( \pm 4.84)\end{array}$ & Never \\
\hline $\begin{array}{r}\text { Time to Assemble } \\
\text { Three Units }\end{array}$ & $\begin{array}{c}11.1 \\
( \pm 3.96)\end{array}$ & $\begin{array}{c}\text { Not yet } \\
\text { attempted }\end{array}$ & $\begin{array}{c}\text { Not yet } \\
\text { attempted }\end{array}$ \\
\hline $\begin{array}{r}\text { Time to Assemble } \\
\text { Four Units }\end{array}$ & $\begin{array}{c}6.9 \\
( \pm 2.08)\end{array}$ & $\begin{array}{c}\text { Not yet } \\
\text { attempted }\end{array}$ & $\begin{array}{c}\text { Not yet } \\
\text { attempted }\end{array}$ \\
\hline \multicolumn{4}{|r}{} \\
\hline \multicolumn{4}{|r}{}
\end{tabular}

\section{B. Bonding Using Fluid Flow}

The concept for the second physical implementation incorporates a new method for bonding that stemmed from experience gained experimenting with the first set of modules. The electromagnetic/permanent magnet bonding mechanism lacked robustness in that it led to improper bonds and weak rejection forces. However, the main weak point of the design is that it is difficult to implement on the microscale due to power constraints and the difficulty of manufacturing electromagnets at such a small scale. The proposed solution overcomes the shortcomings of the first design and provides a method of bonding that can be implemented at the microscale. The major difference between the designs of the first and second modules is that the second utilizes fluid flow to provide the bonding force. The electromagnetic/permanent magnet bonding mechanism is replaced by a face with orifices to allow fluid to flow into the cube where it is controlled by a valve. Each side consists of this orifice/valving interface and internally all valves are connected to a common chamber. Figures $5 \mathrm{a}$ and 5 b show a sample unit.

The experimental environment contains a substrate with a face that is compliant to the face of the modules. The substrate acts as a sink were fluid exits to an external pump and then forces the fluid back into the system at various locations to provide energy for agitation of the modules. The force of the fluid moving toward the sink attracts the modules to the substrate. The module determines how the structure grows by choosing the state of its valves, once attached to the substrate: open or closed. The faces that have open valves act as a sink and other modules are then attracted and the structure grows using this method. The force of bonding is therefore the pressure drop through the module multiplied by the area of the face. Figure $5 \mathrm{c}$ illustrates the concept of this robot system.

1) Experiment III: Self-Assembly and Self-Reconfiguration: The experiment runs similar to the first set of experiments. One module is set on the substrate and the other is set at some distance from it in the fluid. When the second module attaches to the first they determine they have reached the first configuration. The module on the substrate then begins the reconfiguration stage by opening another face's valve and closing the valve on the side where the second cube attached. The experiment completes when the second modules floats around and attaches at the new configuration point.

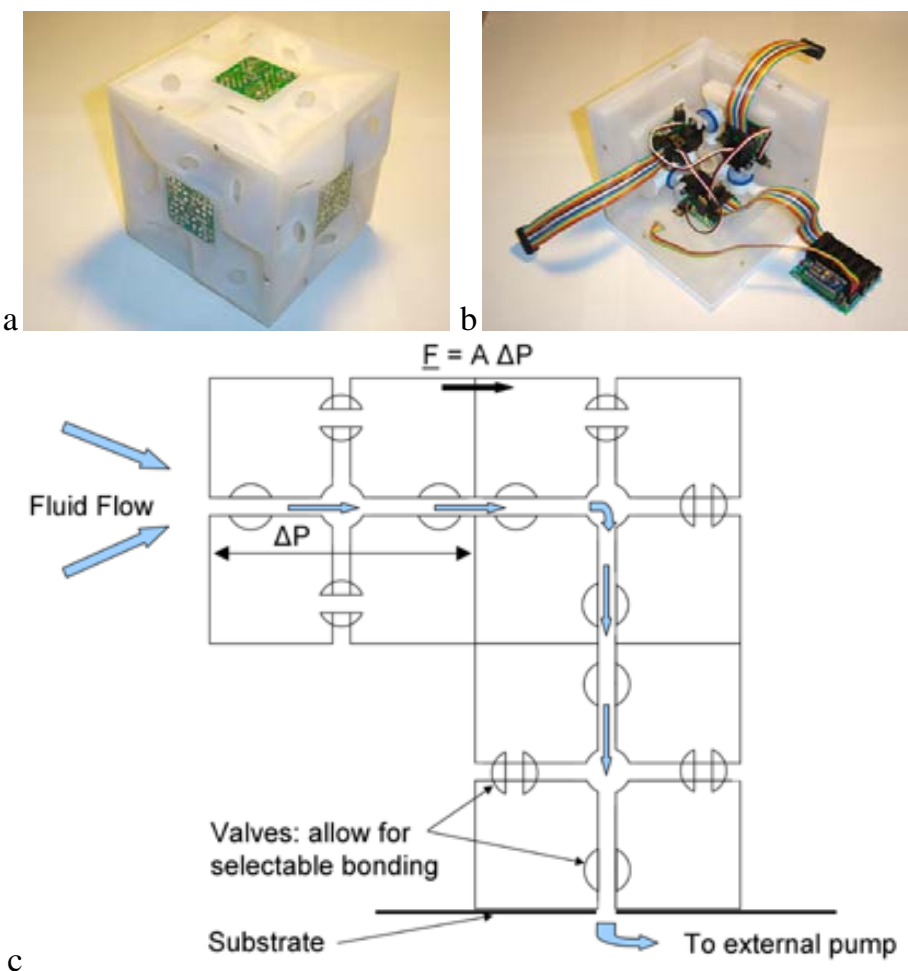

Fig. 5. Overview of the fluid flow bonding robot system. a: The modules' exteriors are equipped with the fluid distribution manifold and the hermaphroditic electrical connector. The geometry of the manifold ensures proper module alignment with the substrate. b: The modules' interiors contain the valves allowing selectable bonding, the central manifold and the robot controller. c: 2D illustration of the fluidic bonding mechanism. The dormant floating module is attracted to the substrate by the fluid flow: when attracted and aligned, it powers up and coordinates with the substrate which valves to open to catalyze pre-programmed structure growth.

\section{DisCUSSION AND CONCLUSIONS}

In this paper we have introduced the first physical, three-dimensional stochastic robot systems. We have demonstrated that both systems we introduced are capable of both self-assembly -in which an anchored structure is built up from successive bonding of free-floating units-and reconfiguration-in which units are both released and rebonded to form a different structure. Unlike all previous approaches to physical modular robotics, the individual units are not powered when not attached to the growing structure, nor can they move on their own. Random motion induced by the surrounding medium, together with selective bonding orchestrated by the growing structure, allow for deterministic self-assembly and reconfiguration. Furthermore, we have shown that the mean time to bonding of units is dependent on the density of units in the medium, and that there is some range of system energy which minimizes time to bonding. The second physical implementation, which only relies on directed fluid flow for bonding and detachment, is composed of technologies that could be scaled down to the microscale, thereby opening up the possibility of constructing a selfassembling, self-reconfigurable robot system at that scale.

In simulation, it is possible to work with many more units (a total of 300), and we have here demonstrated that the 

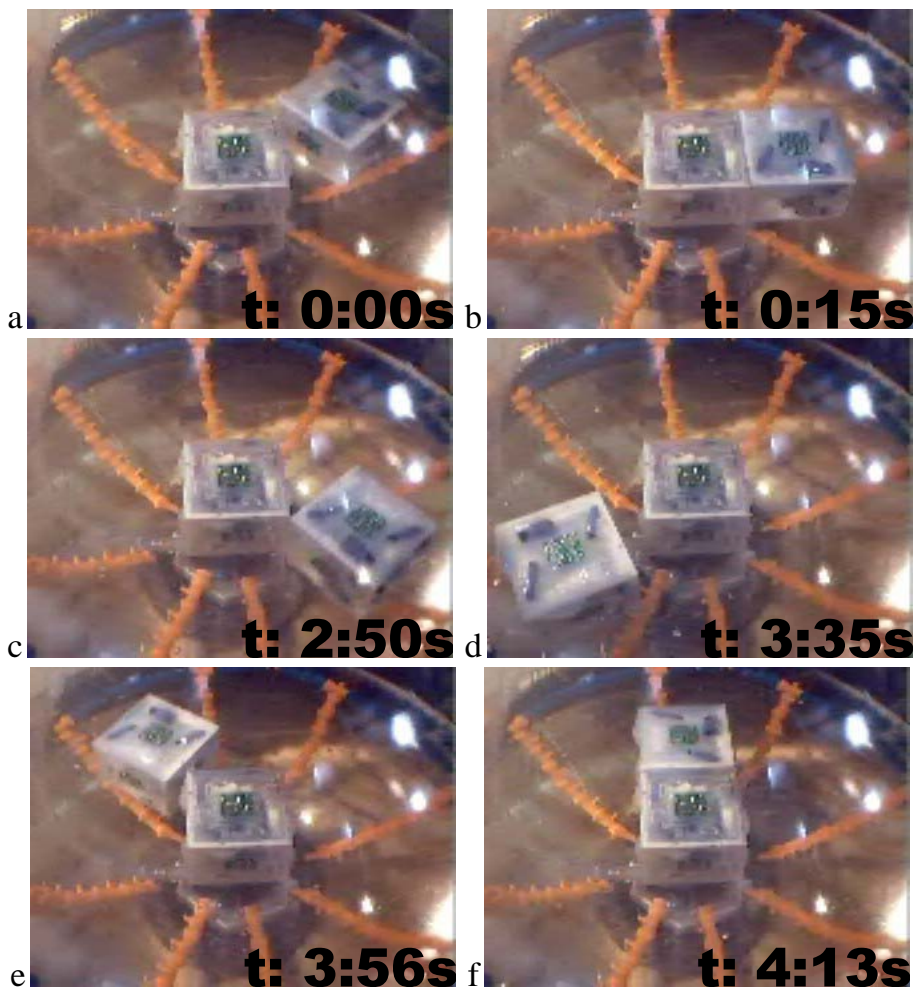

Fig. 6. Physical self-assembly and reconfiguration using fluid flow for bonding. a: Module 1 (central) is attached to the substrate beneath it. module 2 (right-hand) floats dormantly in the fluid. b: Module 1 intakes in fluid through its right-hand manifold, thus attracting module 2. c: Upon detecting module 2 attached to its right-hand manifold, module 1 re-directs the fluid intake to its rear manifold. This causes module 2 to detach from module 1 . d,e: Module 2 floats dormantly, driven by the fluid flow in the tank. f: Module 2 bonds to the rear manifold of module 1 .

total time to assemble a complex structure is dependent not only on density of units, system energy and the strength of unit attraction and retention, but also on an additional factor: the use (or lack of use) of reconfiguration. It was shown that one construction process that involved rejection as well as attraction and retention allowed for faster mean time to completion compared to an alternative construction process for the same shape, but which did not involve any reconfiguration.

These results indicate that three-dimensional self-assembly and reconfiguration is indeed possible in stochastic robot systems. Second, they indicate that the construction process (such as whether to exploit reconfiguration during assembly) is just as important for maximizing the likelihood of realizing a certain structure as the design of the units themselves.

Stochastic robotics holds much promise for the future of micro-robotics. Individual power sources and self locomotion becomes increasingly infeasible for very small and very numerous units, while random motion induced by Brownian motion, and the drawing of power and communication only when anchored to a growing structure, becomes increasingly more realistic. In future work we intend to investigate selfassembly and reconfiguration with more and smaller units, as well as experiment with different unit designs, communication and construction strategies, the use of different fluid mediums such as water, and different methods for system agitation.

\section{REFERENCES}

[1] T. Fukuda and Y. Kawauchi, "Cellular robotic system (CEBOT) as one of the realization of self-organizing intelligent universal manipulator," in IEEE International Conference on Robotics and Automation (ICRA90), 1990, pp. 662-667.

[2] M. Yim, "A reconfigurable modular robot with many modes of locomotion," in JSME Intl. Conf on Advanced Mechatronics, Tokyo, Japan, 1993.

[3] M. Yim, D. Duff, and K. Roufas, "PolyBot: a modular reconfigurable robot," in IEEE International Conference on Robotics and Automation (ICRAO0), 2000, pp. 514-520.

[4] C. Chiang and G. Chirikjian, "Similarity metrics with applications in modular robot motion planning," Autonomous Robots (special issue on Modular Reconfigurable Robots), vol. 10, no. 1, pp. 91-106, 2001.

[5] D. Rus and M. Vona, "Crystalline robots: Self-reconfiguration with compressible units modules," Autonomous Robots (special issue on Modular Reconfigurable Robots), vol. 10, no. 1, pp. 107-124, 2001.

[6] K. Kotay and D. Rus, "Motion synthesis for the self-reconfiguring molecule," in IEEE/RSJ International Conference on Intelligent Robots and Systems, 1998, pp. 843-851.

[7] K. Kotay, D. Rus, M. Vona, and C. McGray, "The self-reconfiguring robotics molecule," in IEEE International Conference on Robotics and Automation (ICRA98), 1998, pp. 424-431.

[8] S. Murata, H. Kurokawa, E. Toshida, K. Tomita, and S. Kokaji, "A 3D self-reconfigurable structure," in IEEE International Conference on Robotics and Automation (ICRA98), 1998, pp. 432-439.

[9] S. Murata, E. Yoshida, K. Tomita, H. Kurokawa, A. Kamimura, and S. Kokaji, "Hardware design of modular robotic system," in IEEE International Conference on Robotics and Automation (ICRAO0), 2000, pp. 2210-2217.

[10] K. Tomita, S. Murata, H. Kurokawa, E. Yoshida, and S. Kokaji, "Selfassembly and self-repair method for a distributed mechanical system," in IEEE Transactions on Robotics and Automation, 1999, pp. 1035-1045.

[11] E. Yoshida, S. Murata, A. Kamimura, K. Tomita, H. Kurokawa, and S. Kokaji, "A motion planning method for a self-reconfigurable modular robot," in IEEE/RSJ International Conference on Intelligent Robots and Systems, 2001, pp. 590-597.

[12] S. Ichikawa, T. Miyamae, and F. Hara, Morpho-functional Machines: The New Species. Tokyo: Springer-Verlag, 2003, ch. Emerging of group formation: morphological configuration of multi-robot system, pp. 195215 .

[13] K. Støy, W.-M. Shen, and P. Will, "On the use of sensors in selfreconfigurable robots," in Proceedings of the Seventh International Conference on the Simulation of Adaptive Behavior, B. Hallam, D. Floreano, J. Hallam, G. Hayes, and J.-A. Meyer, Eds., 2002, pp. 48-57.

[14] A. Castano, A. Behan, and P. Will, "The CONRO modules for selfreconfigurable robots," IEEE Transactions on Mechatronics, vol. 7, no. 4, pp. 403-409, 2002.

[15] M. Jørgensen, E. H. Ostergaard, and H. H. Lund, "Modular ATRON: Modules for a self-reconfigurable robot," in IEEE/RSJ International Conference on Intelligent Robots and Systems, 2004, pp. 2068-2073.

[16] T. Taylor, "A genetic regulatory network-inspired real-time controller for a group of underwater robots," in Proceedings of the Eighth Conference on Intelligent Autonomous Systems (IAS-8), Amsterdam, 2004, pp. 403412 .

[17] M. Yim, Y. Zhang, and D. Duff, "Modular reconfigurable robots, machines that shift their shape to suit the task at hand," IEEE Spectrum Magazine, February 2002, cover article.

[18] R. Jackman, S. Brittain, A. Adams, M. Prentiss, and G. Whitesides, "Design and fabrication of topologically complex, three-dimensional microstructures," Science, vol. 280, pp. 2089-2091, 1998.

[19] E. Winfree, F. Liu, L. A. Wenzler, and N. Seeman, "Design and selfassembly of two-dimensional DNA crystals," Nature, vol. 394, pp. 539544, 1998.

[20] E. Klavins, R. Ghrist, and D. Lipsky, "A grammatical approach to selforganizing robotic systems," IEEE Transactions on Automatic Control, 2005 , under review.

[21] J. Bishop, S. Burden, E. Klavins, R. Kreisberg, W. Malone, N. Napp, and T. Nguyen, "Self-organizing programmable parts," in Proceedings of the IEEE/RSJ Intl. Conf. on Intelligent Robots and Systems (IROS), 2005.

[22] P. White, K. Kopanski, and H. Lipson, "Stochastic self-reconfigurable cellular robotics," in IEEE International Conference on Robotics and Automation (ICRA04). IEEE Press, 2004, pp. 2888-2893. 